\title{
A novel constraint on the Primordial Magnetic Fields using 21-cm line absorption signal
}

\section{Teppei Minoda*}

Department of Physics and Astrophysics, Nagoya University, Nagoya 464-8602, Japan

E-mail: minoda.teppeida.mbox.nagova-u.ac.ip

\section{Hiroyuki Tashiro}

Department of Physics and Astrophysics, Nagoya University, Nagoya 464-8602, Japan

\section{Tomo Takahashi}

Department of Physics, Saga University, Saga 840-8502, Japan

\begin{abstract}
The observation of redshifted 21-cm absorption signal enables us to constrain the intergalactic medium (IGM) gas thermal history. According to the standard scenario of the cosmological structure formation, the sky-averaged (global) $21-\mathrm{cm}$ line signal is expected to be detected as an absorption signal during the Dark Ages. In this paper, we study a possible constraint on the primordial magnetic fields (PMFs) strength with global 21-cm line observation. PMFs can heat up the IGM gas in the Dark Ages due to magnetohydrodynamic energy dissipation mechanisms. The global 21-cm absorption signal can be detected only when the IGM gas is cooler than the cosmic microwave background (CMB) photons. We solve the evolution equations for the IGM gas temperature, ionization fraction and the magnetic field strength, and investigate the global 21$\mathrm{cm}$ signal with the PMFs. Moreover, we demonstrate that, if the redshift of the 21-cm absorption signal is detected at $z \simeq 17$, the PMF strength smoothed on $1 \mathrm{Mpc}$ can be constrained as $B_{1 \mathrm{Mpc}} \lesssim$ $10^{-10}$ Gauss.
\end{abstract}

The 4th KMI International Symposium (KMI2019)

18-20, February 2019

Nagoya, Japan

\footnotetext{
*Speaker.
} 


\section{Introduction}

Thanks to a lot of observations, a wide variety of astronomical objects from planets and stars to galaxies and galaxy clusters are magnetized, but the origin of such magnetic fields remains unknown. One possibility is to create weak magnetic fields in the early universe, and such fields are generally called "Primordial Magnetic Fields (PMFs)". So far, many types of mechanisms have been studied to produce PMFs before the recombination epoch (for detailed reviews, see references [U, [2]). On the other hand, constraints on PMFs are also investigated well. For example, observations of the cosmic microwave background (CMB) have given a constraint on PMFs as $B_{1 \mathrm{Mpc}} \lesssim 4.4 \mathrm{nG}$ [目]. Recently, Saga et al. [䧃] suggested that PMF strength on smaller scale than 1 kpc is strongly constraint by considering the magnetic reheating process, as $B_{1 \mathrm{kpc}} \lesssim 10^{3} \mathrm{nG}$. They investigated the increase of the baryon-to-photon ratio between the era of Big Bang Nucleosynthesis $(\mathrm{BBN})$ and the recombination epoch due to the energy dissipation of the PMFs. If PMFs survive after the recombination epoch, they could also affect the intergalactic medium (IGM) gas considerably [ [D], and its signature is perhaps probed by the 21-cm line observation [ [6].

The purpose of this study is to investigate the thermal history of the IGM gas with the PMF dissipation, and to give a prediction for a constraint on the PMF strength from the global $21-\mathrm{cm}$ line signal. Recently EDGES has reported the global 21-cm absorption signal at $z \sim 17$ was detected $[\square]$. We demonstrate that, if this signal will be confirmed, the global $21-\mathrm{cm}$ signal provides a great impact on the PMF constraint. The detailed calculation methods are described in our previous papers [ [8, 四, 四] and we adopt the flat- $\Lambda$ CDM cosmology with Planck 2015 cosmological parameters [प]] as $h=0.678, \Omega_{\mathrm{c}} h^{2}=0.1186$ and $\Omega_{\mathrm{b}} h^{2}=0.02226$, where $h, \Omega_{\mathrm{c}}, \Omega_{\mathrm{b}}$ are the reduced Hubble constant, and the cold dark matter and baryon density parameters respectively.

\section{Methods}

The global differential brightness temperature at redshift $z$ is given by

$$
\delta T_{b}(z) \propto x_{\mathrm{HI}}(z)\left[1-\frac{T_{\gamma}(z)}{T_{\mathrm{S}}(z)}\right](1+z)^{1 / 2},
$$

where $x_{\mathrm{HI}}$ is the neutral fraction of hydrogen atoms, $T_{\gamma}$ is the background radiation temperature, and $T_{\mathrm{S}}$ is the spin temperature of hydrogen, which is determined by population ratio between two hyperfine levels. After the first stars formed and emitted strong Ly- $\alpha$ photons, $T_{\mathrm{S}}$ is considered to couple with the kinetic gas temperature $T_{\mathrm{K}}$ due to the so-called Wouthuysen-Field effect [[2], [13]]. A detection of the global $21-\mathrm{cm}$ absorption signal suggests that $\delta T_{b}<0$, which means that $T_{\mathrm{K}}$ and $T_{\mathrm{S}}$ are lower than the CMB temperature $T_{\gamma}$.

In this study, we evaluate the cosmological history of $T_{\mathrm{K}}$ with effects of PMF dissipation, and we aim to put a constraint on the PMF models from the $21-\mathrm{cm}$ absorption condition $T_{\mathrm{K}}<T_{\gamma}$. The time evolution of $T_{\mathrm{K}}$ is calculated as

$$
\dot{T}_{\mathrm{K}}(t)=-2 H(t) T_{\mathrm{K}}(t)+\frac{x_{\mathrm{i}}(t)}{1+x_{\mathrm{i}}(t)} \frac{8 \rho_{\gamma}(t) \sigma_{\mathrm{T}}}{3 m_{e} c}\left[T_{\gamma}(t)-T_{\mathrm{K}}(t)\right]+\frac{2}{3 k_{\mathrm{B}} n_{\mathrm{H}}(t)}\left[\dot{Q}_{\mathrm{AD}}(t)+\dot{Q}_{\mathrm{DT}}(t)\right] .
$$

Now we denote the Hubble parameter, ionization fraction of hydrogen, Thomson scattering crosssection, the rest mass of an electron, the speed of light, Boltzmann constant and number density 
of the hydrogen atoms by $H(t), x_{\mathrm{i}}(t), \rho_{\gamma}(t), \sigma_{\mathrm{T}}, m_{e}, c, k_{\mathrm{B}}$, and $n_{\mathrm{H}}(t)$, respectively. The first term is adiabatic cooling from the cosmic expansion, and the second one is heating (or cooling) caused by Compton scattering with CMB photons. The last term represents the PMF heating rate due to ambipolar diffusion (AD) and the decaying turbulence (DT), and given by

$$
\begin{aligned}
& \dot{Q}_{\mathrm{AD}}(t)=\frac{|[\nabla \times \mathbf{B}(t)] \times \mathbf{B}(t)|^{2}}{16 \pi^{2} \xi(t) \rho_{\mathrm{H}}^{2}(t)} \frac{1-x_{\mathrm{i}}(t)}{x_{\mathrm{i}}(t)}, \\
& \dot{Q}_{\mathrm{DT}}(t)=\frac{3 w_{B}}{2} H(t) \frac{|\mathbf{B}(t)|^{2}}{8 \pi} a^{4}(t) \frac{\left[\ln \left(1+t_{d} / t_{\mathrm{rec}}\right)\right]^{w_{B}}}{\left[\ln \left(1+t_{d} / t_{\mathrm{rec}}\right)+\ln \left(t / t_{\mathrm{rec}}\right)\right]^{w_{B}}} .
\end{aligned}
$$

Here, $\xi$ is the drag coefficient of AD and $t_{d}$ is the typical time-scale of DT, and their values are given in [4], [5]]. $\rho_{\mathrm{H}}(t)$ is the hydrogen energy density, $a(t)$ is the cosmic scale factor, and $t_{\mathrm{rec}}$ is the recombination time. We also assume $w_{B}=2\left(n_{B}+3\right) /\left(n_{B}+5\right)$ is constant (see equation (2.5) for the definition of $n_{B}$ ).

To calculate the equations $([2.3)$ and $(\mathbb{R} .4)$, we need the spatial distribution of PMFs. The PMF strength distribution differs from mechanisms of the magnetogenesis. In this paper, we assume that the scale dependence of the PMFs is given by a power-law of coherent wavenumber $k$ as

$$
B_{\lambda}=B_{1 \mathrm{Mpc}}\left(\frac{k}{k_{n}}\right)^{\left(n_{B}+3\right) / 2},
$$

with the spectral index $n_{B}$, and the pivot scale $k_{n}$ is set to $2 \pi \mathrm{Mpc}^{-1}$ here. We mention that PMF is expected to have the smallest scale due to the radiative viscosity in the early universe, and it is approximately given by

$$
\left(\frac{k_{\mathrm{cut}}}{k_{n}}\right)^{n_{B}+5}=1.32 \times 10^{-3}\left(\frac{B_{1 \mathrm{Mpc}}}{1 \mathrm{nG}}\right)^{2} .
$$

Now we can statistically estimate $|\mathbf{B}|$ and $|(\nabla \times \mathbf{B}) \times \mathbf{B}|$ by integrating all scales larger than $k_{\text {cut }}$.

\section{Results and Discussion}

We solve the equation (2.2) for various combinations of $B_{1 \mathrm{Mpc}}$ and $n_{B}$. Because the 21-cm absorption signal can be observed when the kinetic gas temperature $T_{\mathrm{K}}$ is lower than the CMB temperature $T_{\gamma}$, we can put a constraint on PMF models by giving the redshift of the $21-\mathrm{cm}$ absorption signal. Here, we show the resultant PMF constraint for the absorption redshift $z_{\mathrm{abs}}=17.0$ in the figure $\mathrm{W}$. We obtained the upper limit of the PMF normalized amplitude as $B_{1 \mathrm{Mpc}} \lesssim 1.2 \times 10^{-10} \mathrm{G}$ for $n_{B}=-2.9, B_{1 \mathrm{Mpc}} \lesssim 6.3 \times 10^{-12} \mathrm{G}$ for $n_{B}=-2.0$ and $B_{1 \mathrm{Mpc}} \lesssim 2.0 \times 10^{-13} \mathrm{G}$ for $n_{B}=-1.0$. We also plot other constraints on the PMFs in the figure $\mathbb{W}$, which are the CMB anisotropy constraint with the grey-dotted line [3] and the magnetic reheating one with the grey-dashed line [ $[$ ]. As

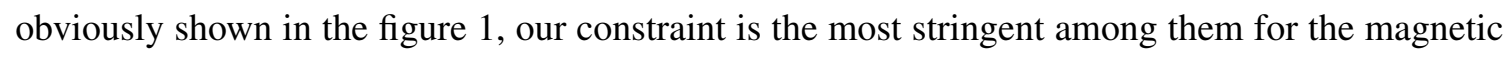
spectral index $-3<n_{B}<-2$.

\section{Summary}

In this work, we investigated the effect of the PMFs on the global 21-cm signal. The PMFs may heat up the IGM gas considerably due to the MHD dissipation mechanisms. When the IGM 


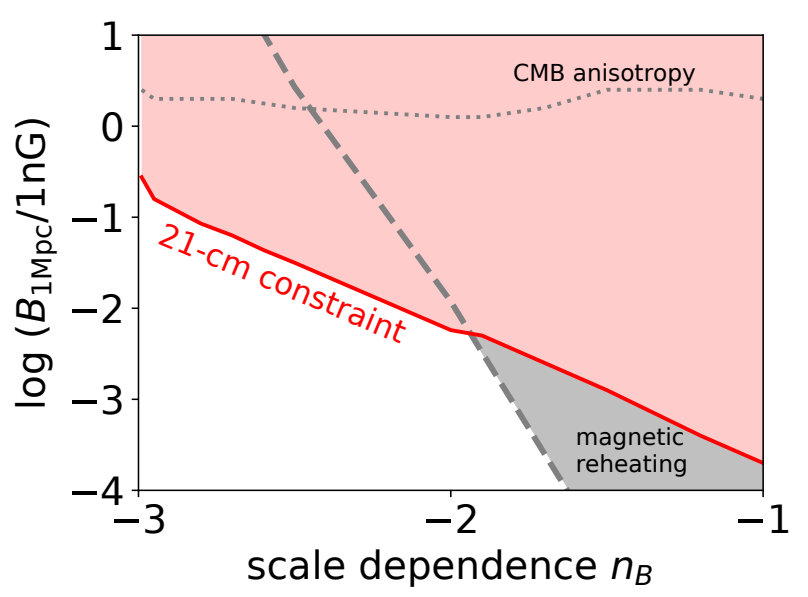

Figure 1: A constraint on the PMF amplitude and scale dependence from the 21-cm line absorption signal. The horizontal and vertical axes give $n_{B}$ and $B_{1 \mathrm{Mpc}}$, the red solid line shows the $21-\mathrm{cm}$ constraint for $z_{\mathrm{abs}}=$ 17.0.

kinetic gas temperature surpasses the CMB temperature, the 21-cm absorption signal is no longer detected. In this study, we numerically solved the thermal history of the IGM gas during the Dark Ages with MHD dissipation effects. Finally we found that the condition $T_{\mathrm{K}}<T_{\gamma}$ at $z=17.0$ gives a strong constraint on the PMFs roughly as $B_{1 \mathrm{Mpc}} \lesssim 10^{-10} \mathrm{G}$.

\section{Acknowledgments}

We appreciate the Organizing Committee of the 4th KMI International Symposium (KMI2019), entitled "Quest for the Origin of Particles and the Universe". We also thank Shohei Saga, Daisuke Yamauchi, Kenji Hasegawa and Hidenobu Yajima for fruitful discussions. This work is supported by KAKEN Grant-in-Aid for Young Scientists (B) Grant Number 15K17646 (HT), JSPS KAKENHI Grant Number 15K05084 (TT), 17H01131 (TT) and MEXT KAKENHI Grant Number 15H05888 (TT).

\section{References}

[1] Kandus, A., Kunze, K. E., \& Tsagas, C. G. 2011, Phys. Rep., 505, 1.

[2] Subramanian, K. 2016, Reports on Progress in Physics, 79, 76901.

[3] Planck Collaboration, Ade, P. A. R., Aghanim, N., et al. 2016, A\&A, 594, A19.

[4] Saga, S., Tashiro, H., \& Yokoyama, S. 2018, MNRAS, 474, L52

[5] Sethi, S. K., \& Subramanian, K. 2005, MNRAS, 356, 778

[6] Sethi, S. K., \& Subramanian, K. 2009, J. Cosmology Astropart. Phys., 11, 021

[7] Bowman, J. D., Rogers, A. E. E., Monsalve, R. A., et al. 2018, Nature, 555, 67.

[8] Minoda, T., Hasegawa, K., Tashiro, H., Ichiki, K., \& Sugiyama, N. 2017, Phys. Rev. D, 96, 123525 
[9] Minoda, T., Tashiro, H., \& Takahashi, T. 2018, arXiv:1812.00730

[10] Minoda, T., Hasegawa, K., Tashiro, H., Ichiki, K., \& Sugiyama, N. 2018, Galaxies, 6, 143

[11] Planck Collaboration, Ade, P. A. R., Aghanim, N., et al. 2016, A\&A, 594, A13.

[12] Wouthuysen, S. A. 1952, AJ, 57, 31

[13] Field, G. B. 1959, ApJ, 129, 536

[14] Schleicher, D. R. G., Banerjee, R., \& Klessen, R. S. 2008, Phys. Rev. D, 78, 083005

[15] Schleicher, D. R. G., Banerjee, R., \& Klessen, R. S. 2009, ApJ, 692, 236 